\title{
A Family of Quadratically-Solvable 5-SPU Parallel Robots
}

\author{
Júlia Borràs, Federico Thomas and Carme Torras
}

\begin{abstract}
A 5-SPU robot with collinear universal joints is well suited to handling an axisymmetric tool, since it has 5 controllable DoFs and the remaining one is a free rotation around the tool. The kinematics of such a robot having also coplanar spherical joints has previously been studied as a rigid subassembly of a Stewart-Gough platform, it being denoted a line-plane component. It was shown that this component has 8 assembly modes corresponding to the roots of a bi-quartic polynomial. Here we identify a whole family of these 5-SPU robots having only 4 assembly modes, which are obtained by solving two quadratic equations. This family is defined by a simple proportionality constraint relating the coordinates of the base and platform attachments. A geometric interpretation of the architectural singularities of this type of robots in terms of conics is provided, which facilitates their avoidance at the design stage. Parallel singularities obey also a neat geometric structure, which permits deriving a cell decomposition of configuration space. Two practical features of these quadratically-solvable robots are the large maneuverability within each connected component and the fact that, for a fixed orientation of the tool, the singularity locus reduces to a plane.
\end{abstract}

Index Terms-Parallel manipulators, Stewart-Gough platforms, robot kinematics, kinematics singularities.

\section{INTRODUCTION}

Over the past half-century, the Stewart-Gough platform has been applied extensively to automate many different tasks due to its well-known merits in terms of speed, rigidity, dynamic bandwidth, accuracy, cost, etc. [1]. There are many important industrial tasks requiring a tool to be perpendicular to a 3D free-from surface along a given trajectory. They include 5-axis milling, laser-engraving, spray-based painting, water-jet cutting, and, in general, any manufacturing task in which the tool is axisymmetric. These tasks can be performed by robots with only 3 translations and 2 rotations; i.e., $5 \mathrm{DoF}$ (degrees of freedom). Since the Stewart-Gough platform has 6 DoF, some limited-DoF parallel robots have been designed for this kind of applications with the aim of simplifying the structure and the control of the general Stewart-Gough platform but without losing its aforementioned merits.

The Stewart-Gough platform consists of a base and a moving platform connected by six UPS (Universal-PrismaticSpherical) legs, where the underline indicates that the prismatic joint is actuated. Thus, it is usually referenced to as a 6-UPS, or equivalently as a 6-SPU, parallel mechanism. If one of these legs is eliminated to obtain a 5-DoF parallel robot, two alternatives arise to make the moving platform

The authors are with the Institut de Robòtica i Informàtica Industrial (CSIC-UPC), Llorens Artigas 4-6, 08028 Barcelona, Spain. E-mails: \{jborras, fthomas, ctorras\}@iri.upc.edu. This work has been partially supported by the Generalitat de Catalunya through the VALTEC program, cofinanced by FEDER funds, and the Spanish Ministry of Education and Science, under the I+D project DPI2007-60858.

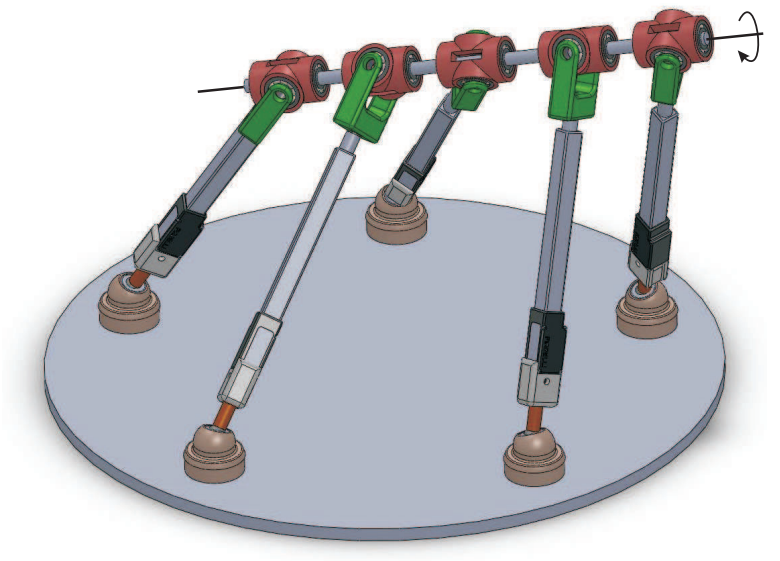

Fig. 1. A 5-SPU parallel robot with aligned universal joints. While the axis defined by these universal joints is rigidly linked to the base for fixed leg lengths, any tool attached to it can freely rotate.

location controllable; namely: (1) adding an extra passive leg, or (2) restraining the mobility of one of the five remaining legs. Then, the challenge consists on how to perform any of these two operations so that the resulting robot has 3 translations and 2 rotations. Y. Zhao and colleagues beat the challenge for the first alternative. They proposed to introduce a PRPU (Prismatic-Revolute-Prismatic-Revolute) passive leg. The properties of the resulting mechanism, technically referenced to as a 5-UPS+PRPU mechanism for obvious reasons, has been analyzed in a series of papers [4], [5], [6]. More recently, Y. Lu and colleagues opted for the second alternative. They proposed a $4 \mathrm{UPS}+\mathrm{S} \underline{P R}$ parallel platform whose static and dynamic properties are studied in [2] and [3], respectively. Many other examples of 5-DoF parallel robots can be found in literature but they greatly depart from the basic 6-UPS design in the sense that they not contain at least 4 UPS legs.

A parallel robot consisting of a base and a moving platform connected by five SPU legs is clearly uncontrollable. For example, if the universal joints are aligned as in Fig. 1 , the moving platform can freely rotate around the axis defined by these five aligned universal joints. Nevertheless, observe that in this particular case the uncontrolled motion is irrelevant if the rotation axis is made coincident with the symmetry axis of the tool. This circumstance might even be advantageous to avoid the entanglement of the wires connected to the tool. In this context, the study of the kinematics properties of 5-SPU parallel robots with coplanar spherical joints and collinear universal joints becomes highly relevant for many applications. Kong and Gosselin refer 
to this particular arrangement of five SPU legs as a lineplane component as it can always be considered as a rigid subassembly in a standard Stewart-Gough platform [11].

Zhang and Song solved, for the first time, the forward kinematics of a general Stewart-Gough platform containing a line-plane component [10]. They showed how the line in the line-plane component of such a platform can have up to eight configurations with respect to the plane and, as a consequence, the platform can have up to 16 assembly modes. The eight configurations of the line correspond to the roots of a bi-quartic polynomial. Therefore, the existence of an algebraic expression for these configurations as a function of the five leg lengths was proved. Husty and Karger studied the conditions for this subassembly to be architecturally singular and found two algebraic conditions that must be simultaneously satisfied [12]. More recently, Borràs and Thomas studied the role of cross-ratios between the location coordinates of the spherical and universal joints centers which will be referred to as attachments in what followsin the characterization of architectural singularities, and in singularity-invariant architectural changes, in line-plane components [9].

Herein we show that, if a simple algebraic relation holds between the base and the platform attachment coordinates of a line-plane component, the number of possible assembly modes drops to 4 so that computing these assembly modes entails calculating the roots of two quadratic polynomials. Moreover, the singularity locus becomes so simple as to permit its straightforward stratification.

The rest of this paper is organized as follows. Section II presents the kinematic and singularity analysis of the general 5-SPU platform, yielding the 8 assembly modes. Section III introduces a family of 5-SPU robots whose forward kinematics can be solved by just computing the roots of two quadratic polynomials. As a consequence, the number of assembly modes drops to 4 and the singularity structure is greatly simplified, leading to a cell decomposition of configuration space, as presented in Section IV. Finally, Section $\mathrm{V}$ points out the extent of the robot family considered as well as some future research directions.

\section{5-SPU Robot with Planar Base and Linear PLATFORM}

Let us consider the 5-legged parallel platform appearing in Fig. 2, whose base and platform attachments lie on plane $\Pi$ and line $\Lambda$, respectively. Let $\Pi$ coincide with the $x y$-plane of the base reference frame. Thus, the leg attachments in the base have coordinates $\mathbf{a}_{i}=\left(x_{i}, y_{i}, 0\right)^{T}$, for $i=1, \ldots, 5$. The pose of $\Lambda$ with respect to $\Pi$ can be described by the position vector $\mathbf{p}=\left(p_{x}, p_{y}, p_{z}\right)^{T}$ and the unit vector $\mathbf{i}=$ $(u, v, w)^{T}$ in the direction of $\Lambda$. Thus, the coordinates of the leg attachments in platform $\Lambda$, expressed in the base reference frame, can be written as $\mathbf{b}_{i}=\mathbf{p}+z_{i} \mathbf{i}$. With this notation, the attachments of the $i$-th leg are determined by the three coordinates $\left(x_{i}, y_{i}, z_{i}\right)$.

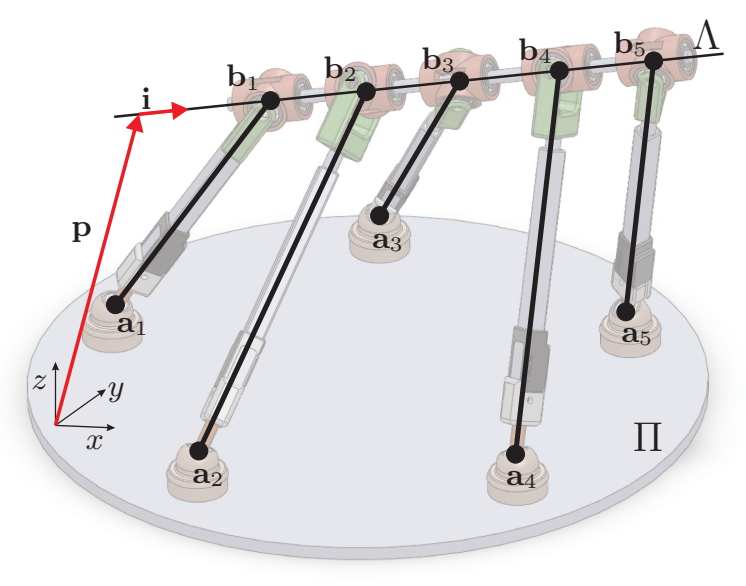

Fig. 2. Schematic representation of the 5-SPU parallel robot in Fig. 1.

\section{A. Singularity Analysis}

It has previously been shown [9] that the Jacobian determinant of a general Stewart-Gough platform containing a lineplane component factors into two terms: one that vanishes when the sixth leg lies on the platform plane, and the other being the determinant of the following matrix:

$$
\mathbf{T}=\left(\begin{array}{cccc}
w p_{z} & w\left(p_{z} u-p_{x} w\right) & w\left(p_{z} v-p_{y} w\right) \\
z_{1} & x_{1} & y_{1} & \\
z_{2} & x_{2} & y_{2} & \\
z_{3} & x_{3} & y_{3} \\
z_{4} & x_{4} & y_{4} \\
z_{5} & x_{5} & y_{5} \\
p_{z}\left(p_{x} w-p_{z} u\right) & p_{z}\left(p_{y} w-p_{z} v\right) & -w^{2} \\
x_{1} z_{1} & y_{1} z_{1} & 1 \\
x_{2} z_{2} & y_{2} z_{2} & 1 \\
x_{3} z_{3} & y_{3} z_{3} & 1 \\
x_{4} z_{4} & y_{4} z_{4} & 1 \\
x_{5} z_{5} & y_{5} z_{5} & 1
\end{array}\right)
$$

which depends exclusively on the 5-legged 5-DoF component.

Thus, the singularity locus of the 5-SPU manipulator studied in this paper corresponds to the root locus of the polynomial resulting from expanding such determinant, i.e.,

$$
\begin{gathered}
C_{1} w p_{z}+C_{2} w\left(p_{z} u-p_{x} w\right)+C_{3} w\left(p_{z} v-p_{y} w\right)+ \\
C_{4} p_{z}\left(p_{x} w-p_{z} u\right)+C_{5} p_{z}\left(p_{y} w-p_{z} v\right)-C_{6} w^{2}=0,
\end{gathered}
$$

where $C_{i}$, for $i=1, \ldots 6$, is the cofactor of the $(1, i)$ entry of $\mathbf{T}$, which depends only on leg attachments.

Architectural singularities occur when all the cofactors are zero, $C_{i}=0$, for $i=1, \ldots 6$.

\section{B. Forward Kinematics}

Similarly to [10], the forward kinematics of our 5-legged parallel robot can be solved by writing the leg lengths as $l_{i}=\left\|\mathbf{b}_{i}-\mathbf{a}_{i}\right\|$, for $i=1, \ldots, 5$. Then, subtracting from the expression for $l_{i}^{2}, i=1, \ldots, 5$, the equation $\|\mathbf{i}\|=u^{2}+v^{2}+$ 
$w^{2}=1$, quadratic terms in $u, v$ and $w$ cancel out yielding

$$
\begin{array}{r}
z_{i} t-x_{i} p_{x}-y_{i} p_{y}-x_{i} z_{i} u-y_{i} z_{i} v \\
+\frac{1}{2}\left(p_{x}^{2}+p_{y}^{2}+p_{z}^{2}+x_{i}^{2}+y_{i}^{2}+z_{i}^{2}-l_{i}^{2}\right)=0,
\end{array}
$$

for $i=1, \ldots, 5$, where $t=\mathbf{p} \cdot \mathbf{i}$.

Subtracting the third equation from the others, quadratic terms in $p_{x}, p_{y}$ and $p_{z}$ cancel out as well. Then, the resulting system of equations can be written in matrix form as

$$
\begin{gathered}
\left(\begin{array}{cccc}
x_{1}-x_{3} & y_{1}-y_{3} & x_{1} z_{1}-x_{3} z_{3} & y_{1} z_{1}-y_{3} z_{3} \\
x_{2}-x_{3} & y_{2}-y_{3} & x_{2} z_{2}-x_{3} z_{3} & y_{2} z_{2}-y_{3} z_{3} \\
x_{4}-x_{3} & y_{4}-y_{3} & x_{4} z_{4}-x_{3} z_{3} & y_{4} z_{4}-y_{3} z_{3} \\
x_{5}-x_{3} & y_{5}-y_{3} & x_{5} z_{5}-x_{3} z_{3} & y_{5} z_{5}-y_{3} z_{3}
\end{array}\right)\left(\begin{array}{c}
p_{x} \\
p_{y} \\
u \\
v
\end{array}\right) \\
=\left(\begin{array}{c}
\left(z_{1}-z_{3}\right) t+N_{1} \\
\left(z_{2}-z_{3}\right) t+N_{2} \\
\left(z_{4}-z_{3}\right) t+N_{4} \\
\left(z_{5}-z_{3}\right) t+N_{5}
\end{array}\right), \quad \text { (4) }
\end{gathered}
$$

where

$$
N_{i}=\frac{1}{2}\left(x_{i}^{2}+y_{i}^{2}+z_{i}^{2}-l_{i}^{2}-x_{3}^{2}-y_{3}^{2}-z_{3}^{2}+l_{3}^{2}\right) .
$$

Now, using simple row/column operations, the determinant associated with the linear system (4) can be written as

$$
\left|\begin{array}{lllll}
x_{1} & y_{1} & x_{1} z_{1} & y_{1} z_{1} & 1 \\
x_{2} & y_{2} & x_{2} z_{2} & y_{2} z_{2} & 1 \\
x_{3} & y_{3} & x_{3} z_{3} & y_{3} z_{3} & 1 \\
x_{4} & y_{4} & x_{4} z_{4} & y_{4} z_{4} & 1 \\
x_{5} & y_{5} & x_{5} z_{5} & y_{5} z_{5} & 1
\end{array}\right|,
$$

which coincides with $C_{1}$ in (2). If (6) vanishes, either $p_{x}, p_{y}, u$, or $v$, can be chosen as parameter, instead of $t$, to reformulate the linear system (4). Since for a nonarchitecturally singular robot not all cofactors are zero, it can be shown that a non-singular linear system of the form (4) can always be found by choosing either $t, p_{x}, p_{y}, u$, or $v$ as parameter.

Solving (4) by Crammer's rule yields

$$
\begin{aligned}
p_{x} & =\left(-C_{2} t+E_{2}\right) / C_{1}, \\
p_{y} & =\left(-C_{3} t+E_{3}\right) / C_{1}, \\
u & =\left(-C_{4} t+E_{4}\right) / C_{1}, \\
v & =\left(-C_{5} t+E_{5}\right) / C_{1},
\end{aligned}
$$

where $E_{i}$ results from substituting the $(i-1)$ th column vector of $C_{1}$ by $\left(N_{1}, \ldots, N_{5}\right)^{T}$.

From equation $u^{2}+v^{2}+w^{2}=1$ and equation (3) for $i=3$, it can be concluded that:

$$
\begin{aligned}
p_{z}^{2} w^{2} & =\left(1-u^{2}-v^{2}\right) \\
& {\left[2\left(-z_{3} t+x_{3} p_{x}+y_{3} p_{y}+z_{3} y_{3} v+z_{3} x_{3} u\right)\right.} \\
& \left.-p_{x}^{2}-p_{y}^{2}-x_{3}^{2}-y_{3}^{2}-z_{3}^{2}+l_{3}^{3}\right] .
\end{aligned}
$$

One the other hand, from $t=\mathbf{p} \cdot \mathbf{i}$,

$$
\left(p_{z} w\right)^{2}=\left(t-p_{x} u-p_{y} v\right)^{2} .
$$

Equating the right hand sides of equations (8) and (9), the following polynomial in $t$ is finally obtained:

$$
n_{4} t^{4}+n_{3} t^{3}+n_{2} t^{2}+n_{1} t+n_{0}=0,
$$

where $n_{4}=-\frac{\left(C_{4} C_{3}-C_{2} C_{5}\right)^{2}}{C_{1}^{4}}$ and

$$
\begin{aligned}
n_{3} & =-\frac{2}{C_{1}^{4}}\left(C_{1}^{2}\left(C_{5} C_{3}+C_{4} C_{2}\right)\right. \\
& +C_{1}\left(C_{5}^{2}+C_{4}^{2}\right)\left(C_{2} x_{3}+\left(C_{1}+C_{4} x_{3}+C_{5} y_{3}\right) z_{3}+y_{3} C_{3}\right) \\
& \left.+\left(C_{4} C_{3}-C_{5} C_{2}\right)\left(E_{5} C_{2}+E_{2} C_{5}-E_{4} C_{3}-E_{3} C_{4}\right)\right)
\end{aligned}
$$

must simultaneously vanish for the forward kinematics of the proposed manipulator to become quadratically-solvable.

Each of the four roots of (10) determines a single value for $p_{x}, p_{y}, u$, and $v$ through (7) and two sets of values for $p_{z}$ and $w$ by simultaneously solving $\|\mathbf{i}\|=1$ and $t=\mathbf{p} \cdot \mathbf{i}$. Thus, up to 8 assembly modes are obtained for a given set of leg lengths.

Finally, note that, if $C_{4}=C_{5}=0$, then $n_{4}=n_{3}=0$. Under this circumstance, the maximum simplification of the forward kinematics of the analyzed robot is obtained: the maximum number of assembly modes drops to 4 . This is discussed in the next section where a family of parallel robots satisfying this condition is studied in detail.

\section{A FAMILY OF QUADRATICALLY-SOLVABLE 5-SPU ROBOTS}

Let us consider the 5-S PU parallel robot whose leg attachment coordinates are $\mathbf{a}_{i}=\left(x_{i}, y_{i}, 0\right)$ and $\mathbf{b}_{i}=\mathbf{p}+z_{i} \mathbf{i}$, with $\mathbf{p}=\left(p_{x}, p_{y}, p_{z}\right)$ and $\mathbf{i}=(u, v, w)$ as before, and subject to the constraint that

$$
z_{i}=\delta x_{i},
$$

where $\delta$ is, thus, a proportionality factor between platform attachments and the $x$-coordinates of the base attachments. To ease readability of the equations, we set $x_{3}=y_{3}=0$ without losing generality. Then $\delta, x_{i}$ and $y_{i}, i=1,2,4,5$, are left as parameters that characterize the family of 5-SPU robots analyzed in this section.

\section{A. Forward Kinematics}

With the attachment coordinates given in (11), the cofactors of the elements of the first row of $\mathbf{T}$ are:

$$
\begin{aligned}
& C_{1}=\delta^{2} F, \\
& C_{2}=-\delta^{3} F, \\
& C_{3}=C_{4}=C_{5}=C_{6}=0,
\end{aligned}
$$

where $F$ can be written as

$$
F=\left|\begin{array}{llll}
x_{1}^{2} & x_{1} y_{1} & x_{1} & y_{1} \\
x_{2}^{2} & x_{2} y_{2} & x_{2} & y_{2} \\
x_{4}^{2} & x_{4} y_{4} & x_{4} & y_{4} \\
x_{5}^{2} & x_{5} y_{5} & x_{5} & y_{5}
\end{array}\right|
$$

and the coefficients of polynomial (10) are:

$$
\begin{aligned}
& n_{4}=n_{3}=0 \\
& n_{2}=\frac{\left(\delta^{2}+1\right) \delta^{2} F^{2}-2 \delta F E_{4}-E_{5}^{2}}{\delta^{2} F^{2}} \\
& n_{1}=2 \frac{E_{2} \delta^{4} F^{2}-F \delta\left(E_{4} E_{2}+E_{5} E_{3}\right)-E_{5}\left(E_{2} E_{5}-E_{3} E_{4}\right)}{\delta^{5} F^{3}} \\
& n_{0}=\frac{\left(E_{2}^{2}+E_{3}^{2}+l_{3}^{2}\left(E_{4}^{2}+E_{5}^{2}\right)\right) F^{2} \delta^{4}-\left(E_{2} E_{5}-E_{4} E_{3}\right)^{2}}{\delta^{8} F^{4}}-l_{3}^{2}
\end{aligned}
$$



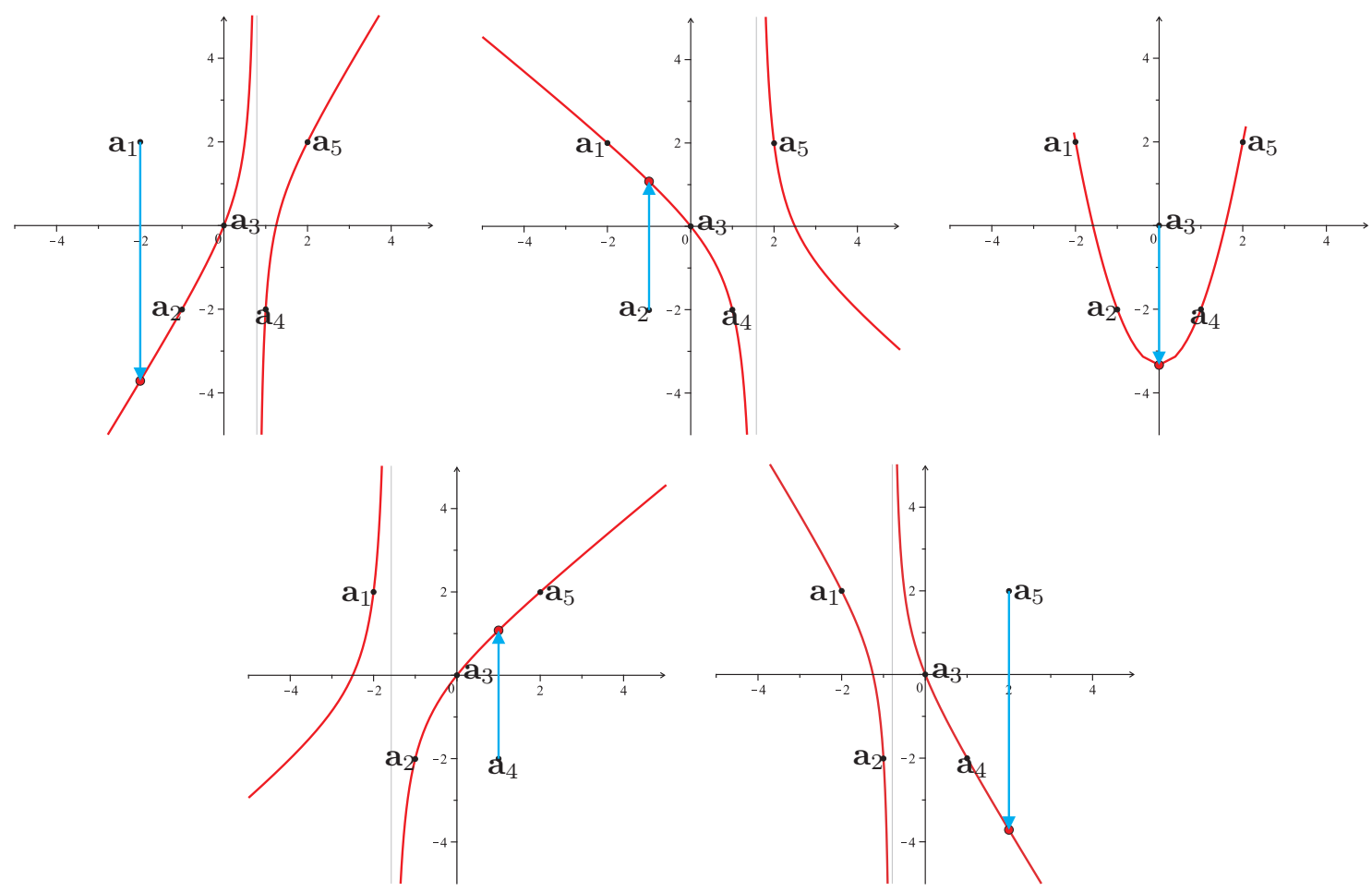

Fig. 3. An attachment should not be located on the conic defined by the other four attachments and the point at infinity $p_{\infty}$ as the platform would then become architecturally singular. In this example the attachments are $\mathbf{a}_{1}=(-2,1), \mathbf{a}_{2}=(-1,-2), \mathbf{a}_{4}=(1,-2)$ and $\mathbf{a}_{5}=(2,2)$, with corresponding $z_{i}=\frac{1}{2} x_{i}$ for $\mathrm{i}=1, \ldots, 5$ (i.e., $\left.\delta=\frac{1}{2}\right)$.

Then, polynomial (10) becomes quadratic and, as a consequence, its two roots can be simply expressed as:

$$
\begin{aligned}
t= & \frac{1}{\delta^{3} F\left(2 \delta F E_{4}+E_{5}^{2}-\left(\delta^{2}+1\right) \delta^{2} F^{2}\right)} \\
& \cdot\left[\delta^{4} F^{2} E_{2}-\delta F\left(E_{2} E_{4}+E_{5} E_{3}\right)\right. \\
& \left.+E_{5}\left(E_{3} E_{4}-E_{2} E_{5}\right) \pm \sqrt{\Delta}\right],
\end{aligned}
$$

where the discriminant is

$$
\begin{aligned}
\Delta= & \delta F\left(E_{5}^{2}+E_{4}^{2}-\delta^{4} F^{2}\right) \\
& {\left[2 \delta^{4} F^{2} E_{4} l_{3}^{2}+\delta^{3} F\left(E_{5}^{2} l_{3}^{2}+E_{3}^{2}\right)+\delta F\left(E_{2}^{2}+E_{3}^{2}\right)\right.} \\
& \left.-\left(\delta^{2}+1\right) \delta^{5} F^{3} l_{3}^{2}+2 E_{3}\left(E_{2} E_{5}-E_{4} E_{3}\right)\right] .
\end{aligned}
$$

Each of the two above roots, say $t_{1}$ and $t_{2}$, determines a single value for $p_{x}, p_{y}$, $u$, and $v$ through (7) and two sets of values for $p_{z}$ and $w$ by simultaneously solving $\|\mathbf{i}\|=1$ and $t=\mathbf{p} \cdot \mathbf{i}$. The resulting four assembly modes are explicitly given by:

$$
\mathbf{p}=\left(\begin{array}{c}
\frac{\delta^{3} F t_{i}+E_{2}}{\delta^{2} F} \\
\frac{E_{3}}{\delta^{2} F} \\
\pm \frac{\left(E_{4}-\delta F\right) \delta^{3} F t_{i}+E_{4} E_{2}+E_{5} E_{3}}{\delta^{2} F \sqrt{\delta^{4} F^{2}-E_{5}^{2}-E_{4}^{2}}}
\end{array}\right)
$$

and

$$
\mathbf{i}=\left(\begin{array}{c}
\frac{E_{4}}{\delta^{2} F} \\
\frac{E_{5}}{\delta^{2} F} \\
\pm \frac{\sqrt{\delta^{4} F^{2}-E_{5}^{2}-E_{4}^{2}}}{\delta^{2} F}
\end{array}\right) .
$$

\section{B. Singularity Analysis}

Substituting the values of the cofactors (12) into (2), the singular configurations of the studied 5-SPU platform are the solutions of the following equation

$$
\delta^{2} w F\left(\delta p_{x} w-(u \delta-1) p_{z}\right)=0 .
$$

Observe that, except for $\delta$, all other design parameters are embedded in $F$, whereas the robot pose appears only in the remaining two factors. Thus, if $F=0$, the manipulator is architecturally singular, i.e., it is always singular independently of its leg lengths. In turn, non-architecturally singular manipulators will reach a singular configuration whenever the other factors nullify. Below we give a geometric interpretation of these two types of singularities, architectural and parallel.

Any set of five points on a plane defines a conic; the one defined by the five base attachments can be expressed, in 
homogeneous coordinates $(x, y, s)_{H}$, as:

$$
\mathcal{C}=\left\{(x, y, s)|| \begin{array}{cccccc}
x^{2} & x y & y^{2} & s x & s y & s^{2} \\
x_{1}^{2} & x_{1} y_{1} & y_{1}^{2} & x_{1} & y_{1} & 1 \\
x_{2}^{2} & x_{2} y_{2} & y_{2}^{2} & x_{2} & y_{2} & 1 \\
0 & 0 & 0 & 0 & 0 & 1 \\
x_{4}^{2} & x_{4} y_{4} & y_{4}^{2} & x_{4} & y_{4} & 1 \\
x_{5}^{2} & x_{5} y_{5} & y_{5}^{2} & x_{5} & y_{5} & 1
\end{array} \mid=0\right\} .
$$

Then, $F=0$ if and only if $p_{\infty}=(0,1,0)_{H} \in \mathcal{C}$. In other words, if the point $p_{\infty}$ belongs to the conic defined by the five base attachments, the parallel robot is architecturally singular. As a consequence, it is possible to assign any value to $y_{i}$, for $i=1, \ldots, 5$, provided that each $\mathbf{a}_{i}=\left(x_{i}, y_{i}\right)$ does not lie on the conic formed by the other four base attachments and $p_{\infty}$ (see Fig. 3).

In particular, if any four base attachments are collinear, the parallel robot would be architecturally singular because the fifth one and $p_{\infty}$ would define a second line, and two intersecting lines can be seen as a degenerate conic.

Let us now turn to the case $F \neq 0$, and study the parallel singularities of non-architecturally singular manipulators. A singular configuration $\mathbf{p}, \mathbf{i} \in \mathbb{R}^{3} \times S^{2}$, with $\mathbf{p}=\left(p_{x}, p_{y}, p_{z}\right)$ and $\mathbf{i}=(u, v, w)$, is that satisfying either $w=0$ or $\left(\delta w p_{x}-(\delta u-1) p_{z}\right)=0$. The first condition holds for configurations where the platform is parallel to the base plane, while the second relates platform position coordinates $\left(p_{x}, p_{z}\right)$ to orientation coordinates $(u, w)$ through the slope equality $p_{z} / p_{x}=w /(u-1 / \delta)$.

Note that singularities can also be expressed in joint space $\mathbb{R}^{5}$ by using the discriminant (15), whose expression only depends on the leg lengths $l_{i}, \mathrm{i}=1, \ldots, 5$. When $\Delta=0$ the two solutions (14) coincide, yielding a singularity. Note that $\Delta$ also consists of two factors, the first one $E_{5}^{2}+E_{4}^{2}-$ $\delta^{4} F^{2}=0$ corresponds to the condition $w=0$ and the other is equivalent to $\left(\delta w p_{x}-(\delta u-1) p_{z}\right)=0$.

An interesting practical consideration is that, if we fix the orientation of the tool, singularities define a plane in position space:

$$
c_{1} p_{x}+c_{2} p_{z}=0
$$

with $c_{1}=\delta w^{2}$ and $c_{2}=w(1-u \delta)$. For example, if the tool is orthogonal to the base plane, i.e. $(u, v, w)=(0,0,1)$, then the robot will reach a singularity when its position, i.e. $\left(p_{x}, p_{y}, p_{z}\right)$, satisfies:

$$
\delta p_{x}+p_{z}=0
$$

It follows from the above singularity analysis that, for a fixed value of $\delta$, the whole family of non-architecturally singular 5-SPU robots considered have exactly the same singularity locus. In other words, given a member of the family, one can freely move its leg attachments without modifying the singularity locus, provided two constraints are maintained, namely the proportionality between $x_{i}$ and $z_{i}$, and the conics condition above that precludes architecturally singular leg arrangements. In the next section, the common structure of the singularity locus is studied, and the influence of $\delta$ on its topology is analyzed.
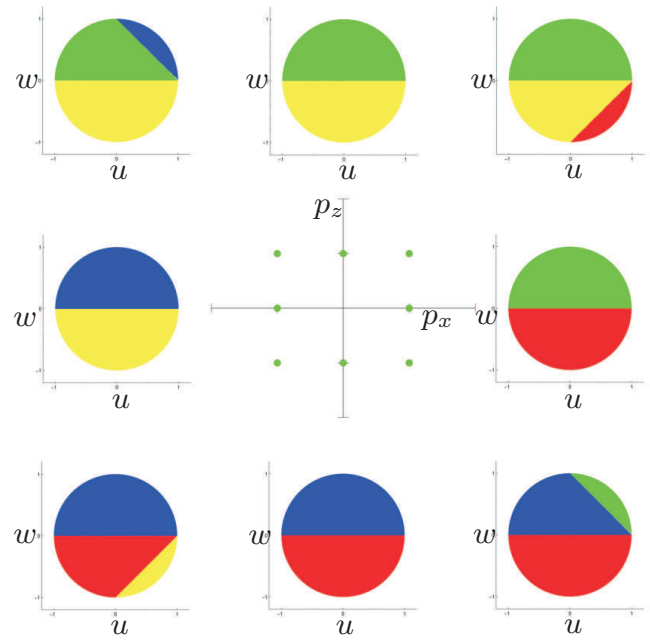

Fig. 4. Representation of the sphere of orientations for eight positions around the origin. The four connected components are marked with different colors.

\section{Cell Decomposition of Configuration Space}

The singularity locus of the 5-SPU robots studied consists of two hypersurfaces in $\mathbb{R}^{3} \times S^{2}$-the robot configuration space (C-space)-, namely:

$$
w=0 \text { and } w p_{x}-\left(u-\frac{1}{\delta}\right) p_{z}=0
$$

Note that, since $p_{y}$ and $v$ do not appear in the hypersurface equations, they do not need to be taken into account when analyzing the topology of singularities. C-space can thus be schematically represented by drawing the sphere of orientations in each point of the plane $p_{x} p_{z}$. Furthermore, only the projection of the sphere in the direction of the $v$ axis needs to be displayed. Figure 4 shows such representation for eight positions around the origin in the plane $p_{x} p_{z}$, for the case $\delta=$ $1^{1}$. Observe that only the relation $p_{z} / p_{x}$ is relevant, therefore each disk stands for all positions in the half-line starting at the origin and having the same $p_{z} / p_{x}$ value. Color encodes where the region lies in relation to the two hypersurfaces. For example, yellow points (the brightest grey level ones) are those where $w<0$ and $w p_{x}-(u-1 / \delta) p_{z}<0$. Lines separating two colors correspond to the two hypersurfaces.

Hence, the two singular hypersurfaces divide $\mathrm{C}$-space into four connected components, corresponding to the four assembly modes in (16) and (17). Note that the symmetry in these equations shows up neatly in the figure. It is worth mentioning that for platform positions in the first quadrant, namely where $p_{x}>0$ and $p_{z}>0$, all the hemisphere of orientations with $w>0$ is reachable. Similarly, there is a whole hemisphere reachable in the other quadrants.

By exploiting the $\mathrm{C}$-space symmetry mentioned above, together with the simplicity of the singular hypersurfaces, it is easy to derive a cell decomposition of C-space. Without

\footnotetext{
${ }^{1}$ The cases $\delta<1$ and $\delta>1$ follow easily from this one, as we will sketch at the end of this section.
} 
going into details, let us just refer to Fig. 5 where a diagram of cells for a single connected component is shown. The $5 \mathrm{D}$ cell is bounded by patches of the two 4D singular hypersurfaces, which intersect at two types of 3D patches: $(w=0, u=1)$ and $\left(w=0, p_{z}=0\right)$. The former corresponds to a fixed orientation of $\Lambda$ perpendicular to the plane $p_{x}=0$ in addition to parallel to $\Pi$ (3 translational DoFs), while the latter corresponds to $\Lambda$ lying on $\Pi$ (3 DoFs in the plane). Two such $3 \mathrm{D}$ patches in turn intersect at a $2 \mathrm{D}$ cell corresponding to $\Lambda$ lying on $\Pi$ and being perpendicular to the plane $p_{x}=0$ (2 translational DoFs). Finally, a 2D cell is bounded by a $1 \mathrm{D}$ cell where, in addition to the preceding conditions, $p_{x}=0$.

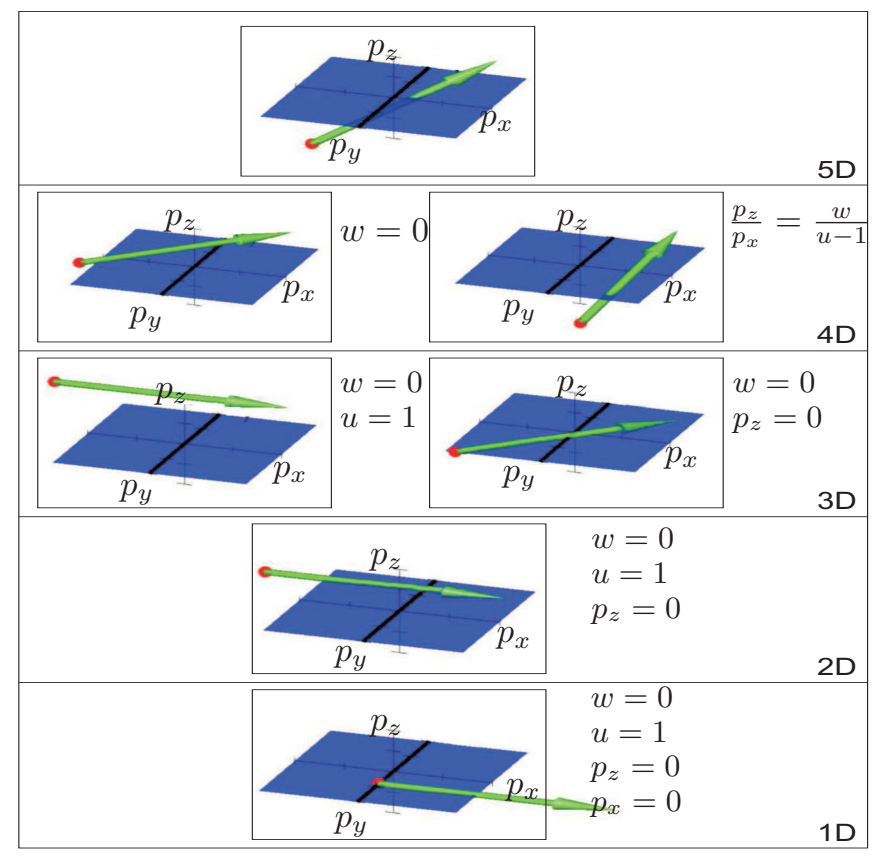

Fig. 5. Diagram showing the relative position of $\Lambda$ and $\Pi$ in each C-space cell.

The four C-space connected components are glued together through some of the lower-dimensional cells, so that the cell decomposition has in total four $5 \mathrm{D}$, six $4 \mathrm{D}$, six $3 \mathrm{D}$, two $2 \mathrm{D}$ and one $1 \mathrm{D}$ cells.

Let us remind that this decomposition is for $\delta=1$. Those for $\delta<1$ and $\delta>1$ can be easily derived by noting that their C-space representations differ only slightly from that in Fig. 4. The four skew line segments standing for the singular hypersurface $(u-1 / \delta) p_{z}-w p_{x}=0$ maintain the same slopes, while their positions vary as a function of $\delta$, so that they intersect hypersurface $w=0$ at $p_{z}=0$ and $u=1 / \delta$ for $\delta>1$, and only at $p_{z}=0$ for $\delta<1$. Consequently, the cell decomposition in the former case has in total four $5 \mathrm{D}$, eight 4D, eight 3D, two $2 \mathrm{D}$ and one $1 \mathrm{D}$ cells, while in the latter case it reduces to four 5D, six 4D, two $3 \mathrm{D}$ and one $2 \mathrm{D}$ cells.

\section{Conclusions}

This paper has presented a family of 5-SPU platforms, with collinear attachments on the platform and coplanar on the base, whose forward kinematics can be solved by computing the roots of two quadratic polynomials, yielding only 4 assembly modes. This important complexity reduction with regards to a general such 5-SPU platform is attained by imposing a simple proportionality relation between the coordinates of the base and platform attachments.

The presented analysis of the 5-SPU robot is useful for the study of 6-SPU Stewart-Gough platforms that contain a lineplane component satisfying the mentioned proportionality relation. The kinematics of such a 6-DoF platform becomes greatly simplified, having a total of 8 assembly modes. A cell decomposition of its singularity locus can be readily derived from that obtained in Section IV, by just considering the additional singular hypersurface corresponding to the platform attachment of the 6th leg lying on the base plane.

This work has also a direct application to the design of reconfigurable robots. It suffices to place base attachments on actuated guides, so as to enlarge the usable workspace or increase platform stiffness, while maintaining the same well-behaved singularity structure.

\section{ACKNOWLEDGEMENT}

The authors thank Patrick Grosch and Albert Sierra for their work on the figures, which led to fruitful discussions.

\section{REFERENCES}

[1] J.-P. Merlet, Parallel Robots, Springer, 2000.

[2] Y. Lu, B. Hu, and J. Xu, "Kinematics analysis and solution of active/passive forces of a 4SPS+SPR parallel machine tool," International Journal of Advanced Manufacturing Technology, Vol. 36, No. 1-2, pp. 178-187, 2008.

[3] Y. Lu and J. Xu, "Simulation solving/modifying velocity and acceleration of a 4UPS+SPR type parallel machine tool during normal machining of a 3D free-form surface," International Journal of Advanced Manufacturing Technology, Vol. 42, No. 7-8, pp. 804-812, 2009.

[4] J. Gao, H. Sun, and Y. Zhao, "The primary calibration research of a measuring limb in 5-UPS/PRPU parallel machine tool," Proc. of the 2004 Int. Conf. on Intelligent Mechatronics and Automation, pp. 304-308.

[5] K. Zheng, J. Gao, and Y. Zhao, "Path control algorithms for a novel 5-DoF parallel machine tool," Proc. of the IEEE Int. Conf. on Mechatronics and Automation, pp. 1381-1385.

[6] Y. Zhao, Y. Hou, Y. Shi, and L. Lu, "Dynamics analysis of a 5UPS/PRPU parallel machine tool," 12th IFToMM World Congress, 2007.

[7] J. Borràs, F. Thomas, and C. Torras, "Architecture Singularities in Flagged Parallel Manipulators," IEEE Intl. Conf. on Robotics and Automation (ICRA), 2008, pp. 3844-3850.

[8] J. Borràs, F. Thomas, E. Ottaviano and M. Ceccarelli, "A Reconfigurable 5-DoF 5-SPU Parallel Platform," ASME/IFToMM Intl. Conf. on Reconfigurable Mechanisms and Robots (ReMAR), London, June 2009.

[9] J. Borràs and F. Thomas, "Kinematics of Line-Plane Subassemblies in Stewart Platforms," IEEE Intl. Conf. on Robotics and Automation (ICRA), 2009, pp. 4094-4099.

[10] C. Zhang and S.M. Song, "Forward Kinematics of a Class of Parallel (Stewart) Platforms with Closed-Form Solutions," Proc. IEEE Intl. Conf. on Robotics and Automation, 1991, pp. 2676-2681.

[11] X. Kong and C.M. Gosselin, "Generation and Forward Displacement Analysis of Two New Classes of Analytic 6-SPS Parallel Manipulators," Journal of Robotic Systems, 2001.

[12] M.L. Husty and A. Karger, "Self-Motions of Griffis-Duffy Type Parallel Manipulators," Proc. IEEE Intl. Conf. on Robotics and Automation, 2000, pp. 7-12. 